\title{
Chylothorax due to Blunt Torso Trauma: A Rare Etiology
}

\section{Annu Babu, Amulya Rattan, Piyush Ranjan, Subodh Kumar and Amit Gupta}

Department of Trauma Surgery and Critical Care, JPN Apex Trauma Centre, All India Institute of Medical Sciences, New Delhi, India

\begin{abstract}
Chylothorax most commonly occurs after thoracic surgery; blunt thoracic trauma is a rare cause of chylothorax. A 50 yr old female presented to our emergency department with history of road traffic injury. On primary assessment the vital parameters were normal and she had a laceration of nasal bridge and lip, with fractures of nasal and right forearm bones. CT scan confirmed fracture of nasal bone, spinous process of D4 vertebrae and bilateral radius. Repair of lip laceration along with open reduction and internal fixation (ORIF) for bilateral radii and nasal bone fracture were done emergently. Patient was allowed orally after 6 hours and recovery seemed to be uneventful. However, patient started complaining of chest heaviness with difficulty in breathing on second post-operative day. On Ultrasound Chest and CT evaluation a radiological diagnosis of traumatic hydrothorax with mediastinal \& retroperitoneal lymphangiectasias was made. Intercostal drains placed bilaterally showed chylothorax. Patient was kept nil per oral and on parenteral nutrition, micronutrient supplementation and adequate analgesia. Empiric antibiotic therapy was started. Intercostal drain output and lipid levels of contents decreased with this regime, gradually enteral fat-restricted diet was started with medium chain triglycerides and patient was allowed full orally from day 6 . Chest drain was removed on day 11 and patient discharged on day 12
\end{abstract}

Keywords: Chylothorax; Torso; Trauma

\section{Introduction}

Chylothorax is accumulation of chyle in pleural cavity [1]. It is most commonly seen after thoracic surgery; blunt trauma is a rare cause $[1,2]$. We are reporting a case of chylothorax due to blunt torso trauma so as to contribute to the scarce body of literature on this rare condition.

\section{Case Report}

A 50 year female presented to the emergency department with history of road traffic injury (bicycle rider hit by a car). On presentation, primary survey and adjuncts (CXR and FAST) were normal. Laceration over nasal bridge and lip, with fractures of nasal bone and bilateral forearm bones were noted. CT scan confirmed fractures of nasal bone, spinous process D4 and bilateral radii. Repair of lip laceration along with ORIF for bilateral radii and nasal bone fracture were done emergently. Patient was kept nil per oral for 6 hours and the recovery seemed to be uneventful.

However, patient started complaining of chest heaviness with breathing difficulty on second post-operative day. Bedside ultrasonography of chest showed $\mathrm{B} / \mathrm{L}$ moderate pleural effusion. Triple contrast enhanced CT scan of chest and abdomen was done which showed fluid hypodensities in the peri-renal areas and mediastinal area suggestive of retroperitoneal and thoracic lymphangiectasias (Figure 1).

Bilateral chest tubes were placed. Right ICD returned opalescent viscous milky content and left was serous (Figure 2). The suspicion of chylothorax was confirmed with biochemical analysis (Table 1).

A diagnosis of traumatic chylothorax with mediastinal \& retroperitoneal lymphangiectasias was thus made. Patient was planned for non operative management and parenteral nutrition was started via central line, keeping the patient nil by mouth. Supportive intravenous fluid therapy was titrated according to urine output. Micronutrient supplementation and adequate analgesia was ensured. Empiric antibiotic therapy was started in view of risk factors (bilateral chest tubes, history of road traffic injury and central line in situ). Daily chest physiotherapy and incentive spirometry were ensured. As the ICD output and lipid levels of ICD contents decreased with this regime (Figure 3), enteral fatrestricted diet was started with medium chain triglycerides and patient was allowed full orally from day 6 .
Pleural fluid and blood cultures were reviewed periodically. Parenteral supplementation was stopped from day 8. Left ICD was removed on day 10 and right on day 11 (Figure 4).

Patient was discharged to home care on day 12. Follow up at 4 and 12 weeks revealed no fresh complaints.

\section{Discussion}

\section{Etiology and differentials}

Chylothorax as a result of trauma is a rare report. Most common cause of chylothorax so far is thoracic surgery (esophageal), in which an incidence of $0.4 \%$ has been reported [3]. Thoracic duct obstruction due to malignancy is the most common cause of non-traumatic chylothorax [2]. Broadly speaking, etiologies of chylothorax are classified as in

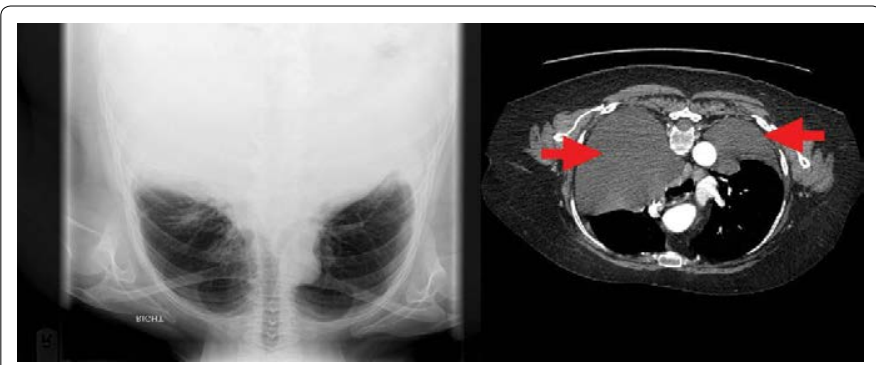

Figure 1: CXR and CECT films.

*Corresponding author: Gupta A, Department of Trauma Surgery and Critical Care JPN Apex Trauma Centre, All India Institute of Medical Sciences, New Delhi, India, Tel: +91 112673 1164; E-mail: amitguptaaiims@gmail.com

Received February 23, 2015; Accepted March 28, 2015; Published March 30, 2015

Citation: Babu A, Rattan A, Ranjan P, Kumar S, Gupta A (2015) Chylothorax due to Blunt Torso Trauma: A Rare Etiology. J Trauma Treat 4: 243. doi: 10.4172/2167-1222.1000243

Copyright: $\odot 2015$ Babu A, et al. This is an open-access article distributed under the terms of the Creative Commons Attribution License, which permits unrestricted use, distribution, and reproduction in any medium, provided the original author and source are credited. 


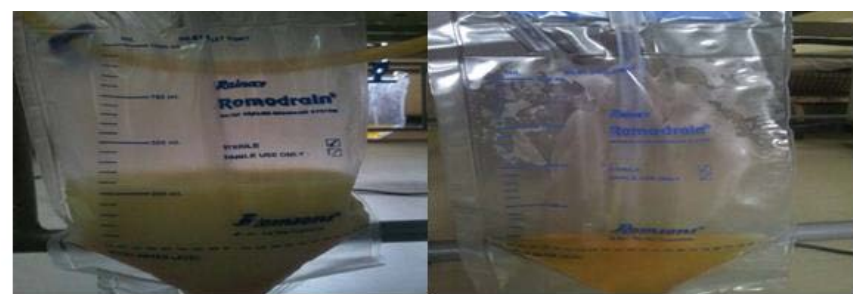

Figure 2: Contents of bilateral ICDs: Right and left respectively.

\begin{tabular}{|l|l|l|l|}
\hline Color & Milky white & Albumin & $\mathbf{1 . 5} \mathbf{~ g / d l ~}$ \\
\hline Triglycerides & $\mathrm{t} 120 \mathrm{mg} / \mathrm{dl}$ & Chylomicrons & ++ \\
\hline Cholesterol & $80 \mathrm{mg} / \mathrm{dl}$ & $\mathrm{pH}$ & 7.4 \\
\hline
\end{tabular}

Table 1: Biochemical analysis of pleural fluid.

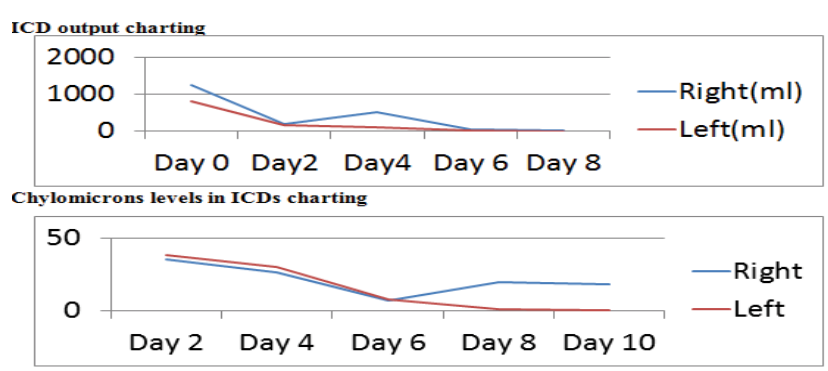

Figure 3: Trends of ICD output.

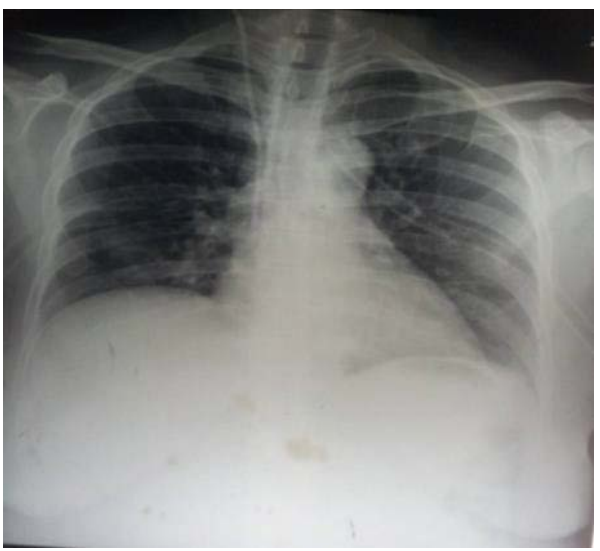

Figure 4: Pre ICD removal CXR.

Figure 5. An entity called hepatic chylothorax has been described in which chylous ascites crosses the diaphragm and accumulates in the pleural space [4]. Pseudochylothorax is defined as exudative effusion remaining in the pleural space for a long period of time (often years) gradually becoming enriched with cholesterol, most common etiologies being tuberculosis, rheumatoid, poorly evacuated empyema and chronic haemothorax [5].

\section{Location}

Bilateral chylothoraces are commoner than unilateral; right (50\%) being commoner than left (33.3\%) [2,3]. The laterality of unilateral effusions are also dependent on the location of the leak [1]. Damage above the fifth thoracic vertebra leads to left sided effusion whereas

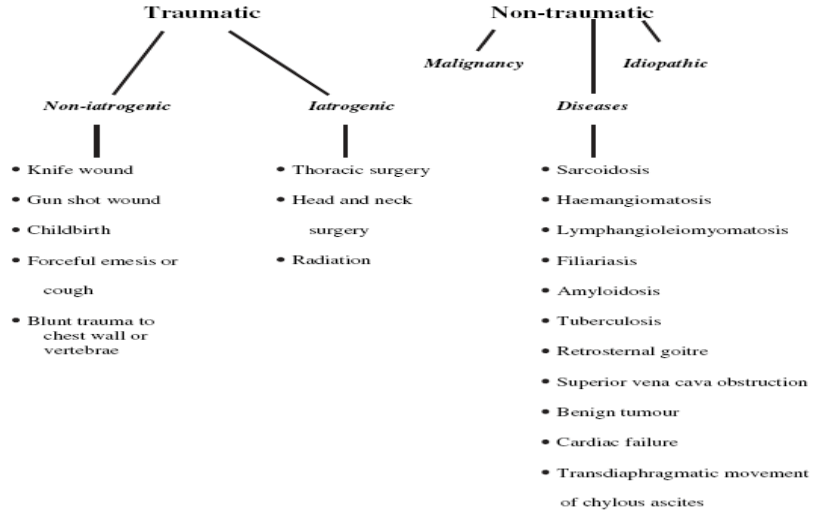

Figure 5: Etiology of chylothorax [16].

damage below this level causes right sided effusion. The volume of effusion also depends upon rate of chyle loss and the concomitant effect of the etiology.

\section{Presentation}

Rapid loss of chyle leads to hypovolaemia and respiratory difficulty as the pleural space fills with fluid besides causing malnutrition due to loss of protein, fats and vitamins. Electrolyte loss leads to hyponatraemia and hypocalcaemia. Opportunistic infections signify significant loss of immunoglobulins, T lymphocytes and proteins into the pleural space. However, they almost never occur in the effusion itself as chyle is bacteriostatic [6]. Persistent drainage via a chest drain in patients with post operative or post-traumatic effusions should be evaluated with a high degree of clinical suspicion, early diagnosis and management is life saving for patient [3].

\section{Diagnosis}

The nature of fluid is classically milky or white in $50 \%$ of cases; however, it may be clear in fasting patients and hemorrhagic in traumatic causes. Confirmation of the diagnosis is by fluid analysis. Lipoprotein analysis demonstrating chylomicrons is the gold standard. Other means of establishing diagnosis are measurement of fluid cholesterol and triglyceride levels. A fluid to serum cholesterol ratio $<1$ and triglyceride ratio $>1$ are highly suggestive. Staats et al. [7] have proposed the following criteria for the biochemical diagnosis of chylothorax:

> Pleural fluid triglyceride of $>110 \mathrm{mg} / \mathrm{dl}: 1 \%$ chance of being nonchylous

> Triglyceride of $<50 \mathrm{mg} / \mathrm{dl}: 5 \%$ chance of being chylous. Chylothorax can be easily confused with empyema due to obvious appearances. Its differentiation from empyema is done by centrifugation; after centrifugation chyle fluid remains uniform whereas a clear supernatant separates in empyema [8]. Differentiation from pseudo-chylothorax is by adding 1-2 ml of ethyl ether; the milky appearance disappears in pseudo-chylothorax [9].

Caution must however be exercised in interpretation of results and they must be interpreted in conjunction with the clinical scenario. Maldonado et al. [10] in a retrospective analysis of 74 patients with chylomicron positive pleural fluid demonstrated that $14 \%$ of the chylous effusions had triglyceride levels less than $110 \mathrm{mg} / \mathrm{dl}$ [10]. The low triglyceride level in all these cases was attributed to perioperative 
fasting or malnutrition. Hence, care should be taken in relying solely on a single criteria for diagnosis.

CT abdomen and thorax should be performed given the strong association with malignancy in cases where pseudo-chylothorax is found [7]. Lymphangiography may be used to demonstrate the site of leakage or blockage.

\section{Treatment}

It can be classified under 3 categories:

> Treatment of the underlying condition

> Non-operative management

> Surgical management.

Nutritional management, empirical antibiotic coverage, adequate analgesia, chest physiotherapy and intensive monitoring are the cornerstones of successful non-operative management. Serial Chest X-rays come handy to look for lung inflation. Nil by mouth or the administration of low to medium chain triglycerides by mouth resolves approximately $50 \%$ of congenital or traumatic chylothoraces [11]. Medium chain triglycerides are directly absorbed into the portal system, bypassing the intestinal lymph system. This reduces the flow of chyle in the thoracic duct allowing it the opportunity to heal [12]. Thoracic duct chyle flow increases after meals especially after a high-fat meal [13]. The volume of chyle flow can be reduced by avoiding fat-containing enteral nutrition [14]. If the chyle leak does not stop following the use of medium chain triglycerides, then total parenteral feeding to reduce the chyle flow even further should be considered $[11,12]$.

Draining large chylothoraces using chest drain insertion are required to ensure complete lung expansion. Monitoring of serum electrolytes, lymphocyte count, albumin and total protein as well as weight are pertinent to care of patients with loss of critical protein and immune cells in patients with chylothorax. Use of somatostatin and octreotide as adjuncts have proved to be useful in the non-operative management of chylothorax. These agents reduce intestinal chyle production, thereby reducing the volume flowing through the injured thoracic duct. Positive attributes of octreotide therapy include, shorter duration of intensive care treatment, reduction of recurrent thoracocentesis, fewer fluid and plasma infusions, reducing the risk of infection. Reported side effects of octreotide are transient changes in blood glucose levels and transient abdominal distension [15]. McGrath et al. found 9 patients that were unlikely to respond to conservative measures in which lymphangiography identified the site of the leak [16]. Leak resolved in all cases and they recommended early lymphangiography in cases unlikely to be cured by conservative methods only.

Indicators of failure of conservative therapy:

> Drains more than $1.5 \mathrm{l} /$ day in an adult or $>100 \mathrm{ml} / \mathrm{kg}$ body weight per day in a child

> Leaks chyle at a rate of $>11$ day for 5 days

> Persistent chyle flow for more than 2 weeks.

> Rapid decline in nutritional status despite conservative management.

Pleurodesis is recommended in malignancy where radiotherapy/ chemotherapy have not led to improvement in the chylo-thorax or the patient is unfit for surgery [17]. Tetracycline, bleomycin and povidone are the agents used for pleurodesis. Pleurectomy or pleurodesis with talc or glue is also indicated in cases where thoracic duct is unidentifiable. Mares et al. have recommended thoracoscopic talc pleurodesis as a safe and highly effective method of palliation in lymphoma related chylothorax refractory to chemotherapy or radiotherapy [18]. Cope et al., also found pleurodesis curative in patients with demonstrable duct leakage [19]. Boffa et al., [20] suggested that pleurodesis is curative in post thoracic surgery chyle leak [20]. Litherland et al., [21] reported that CT guided disruption of the lymphatics had good effect in the management of high output chylothorax [21]. However its reproducibility and success varied in different centres. Cerfolio et al., recommend thoracic duct ligation where duct is suspected to be damaged during surgery [22]. Dougenis et al., advocate prophylactic thoracic duct ligation in all cases of extensive oesophageal resection. In their series of 255 patients, elective ligation of the thoracic duct reduced the incidence of chylothorax from $9 \%$ to $2.1 \%$ [23]. Ligation can be achieved by thoracotomy or thoracoscopy. The main problem remains identifying the chyle leak. Ligation is successful in $90 \%$ of patients when performed just above the right hemi-diaphragm as it halts flow from any unidentified accessory ducts [24]. In case the site of leak is in the region of the neck or upper thorax, ligation is carried out in Poirier's triangle between the arch of the aorta, internal carotid and vertebral column.

In thoracoscopic ligation, a short segment of the duct is excised before clipping the remaining ends. If the leak is not identifiable mass ligation of all the tissue between aorta, spine, esophagus and pericardium is performed. Extensive dissection to find the duct is discouraged as it further increases the risk of trauma and leak.

Pleuroperitoneal shunts are indicated in patients who are unfit for major surgery or have underlying malignancy [25]. It minimises the nutritional or immunological deficits seen in chylothorax.

Urgent thoracic duct ligation is encouraged when duct has been damaged as a complication of surgery. There is a higher mortality in non-operatively managed patients $(50 \%)$ as compared to thoracic duct ligation (10\%) in chylothorax following esophageal surgery. It also reduces the risk of electrolyte disturbance, malnutrition and immunological deficiency [26] (Figure 6) summarizes the management protocol of traumatic chylothorax.

\section{Prognosis}

In the past, the mortality due to chylothorax was in excess of $50 \%$ [15]. Introduction of aggressive therapeutic measures to reverse the adverse effects of chyle loss has led to the lowering of mortality rates for post-traumatic chylothorax.The success of managing large chyle leaks involves aggressive nutritional support and early surgical intervention when indicated. Malignant chylothorax and bilateral chylothoraces have worse prognosis [26].

\section{Conclusion}

Traumatic chylothorax, though a rare entity, can be progressively debilitating and rapidly fatal if timely diagnosis and intervention are not done. Awareness of this rare entity and a high degree of suspicion for unusual pleural effusions are key to successful diagnosis. Non-operative management of traumatic chylothorax is a viable option provided the vital tenets of adequate nutritional support, restricted fatty diet and intensive supportive care are kept in mind. 
Citation: Babu A, Rattan A, Ranjan P, Kumar S, Gupta A (2015) Chylothorax due to Blunt Torso Trauma: A Rare Etiology. J Trauma Treat 4: 243. doi:10.4172/2167-1222.1000243

Page 4 of 5
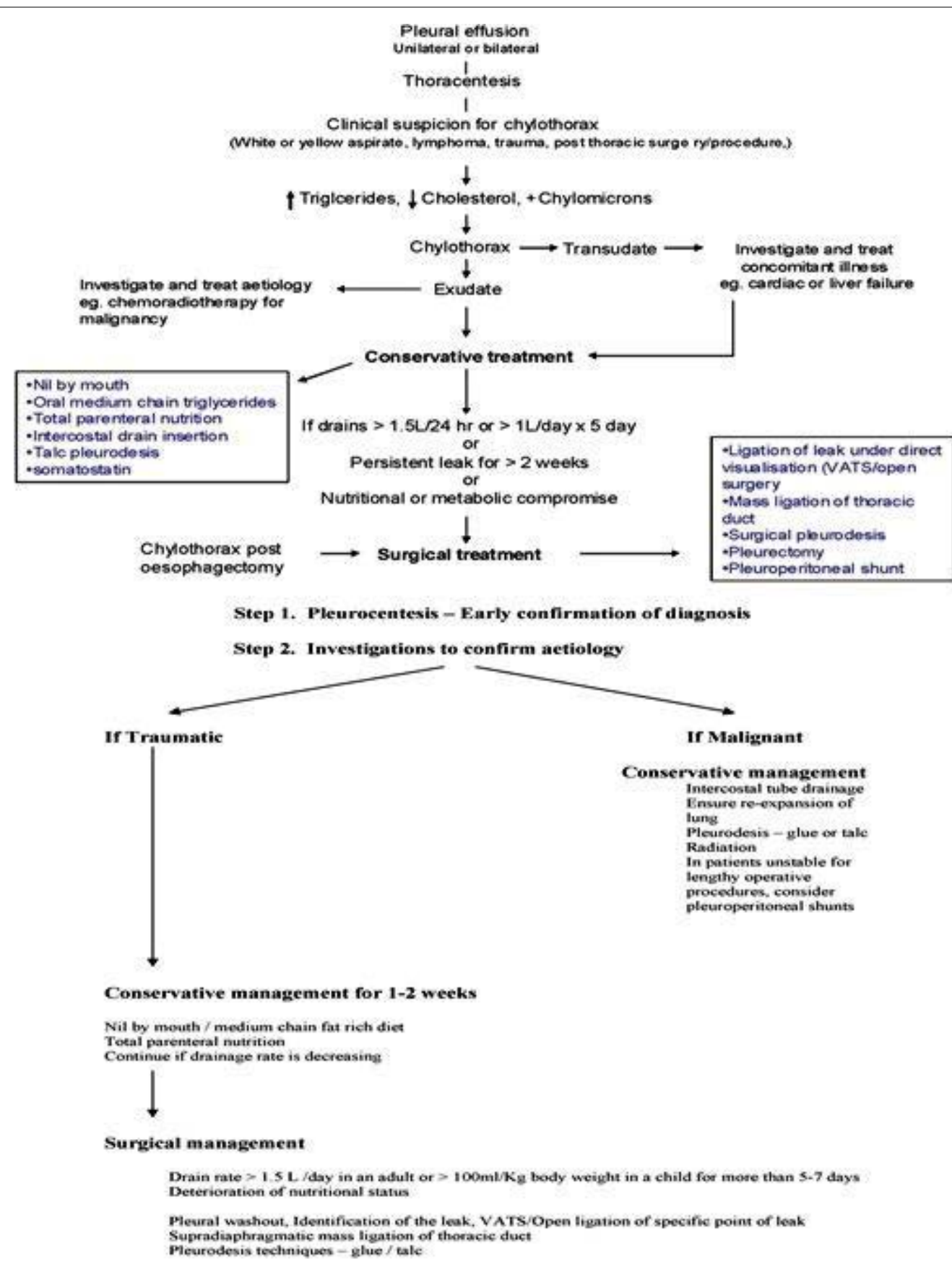

Figure 6: Algorithm for management of suspected traumatic chylothorax [16].

\section{References}

1. Bulent KMD, Gultekin G, Koray D, Unal S (2008) Chylothorax Resulting from a Penetrating Injury of the Neck: a Case Report. European Journal of Trauma and Emergency Surgery 34: 299-301.

2. Schild HH, Strassburg CP, Welz A, Kalff J (2013) Treatment Options in Patients with Chylothorax. Dtsch Arztebl Int 110: 819-826.

3. Doerr CH, Allen MS, Nichols FC 3rd, Ryu JH (2005) Etiology of chylothorax in 203 patients. Mayo Clin Proc 80: 867-870.

4. Nair SK, Petko M, Hayward M P (2007) Aetiology and management of chylothorax in adults. Eur J Cardiothorac Surg 32: 362-369.
5. Hillerdal G (1997) Chylothorax and pseudo-chylothorax. Eur Respir J 10: 1157 1162.

6. Wang JT, Hsueh PR, Sheng WH, Chang SC, Luh KT (2000) Infected chylothorax caused by Streptococcus agalactiae: a case report. J Formos Med Assoc 99: 783-784.

7. Staats BA, Ellefson RD, Budahn LL, Dines DE, Prakash UB, et al. (1980) The lipoprotein profile of chylous and nonchylous pleural effusions. Mayo Clin Proc 55: 700-704.

8. McGrath EE, Anderson PB (2011) Diagnosis of pleural effusion: a systematic approach. Am J Crit Care 20: 119-127.

9. Sassoon CS, Light RW (1985) Chylothorax and pseudo-chylothorax. Clin Chest 
Citation: Babu A, Rattan A, Ranjan P, Kumar S, Gupta A (2015) Chylothorax due to Blunt Torso Trauma: A Rare Etiology. J Trauma Treat 4: 243. doi:10.4172/2167-1222.1000243

Page 5 of 5

Med 6: 163-171.

10. Maldonado F, Cartin-Ceba R, Hawkins FJ, Ryu JH (2010) Medical and surgical management of chylothorax and associated outcomes. Am J Med Sci 339: 314318.

11. Fernandez Alvarez JR, Kalache KD, Grauel EL (1999) Management of spontaneous congenital chylothorax: oral medium-chain triglycerides versus total parenteral nutrition. Am J Perinatol 16: 415-420.

12. De Beer HG, Mol MJ, Janssen JP (2000) Chylothorax. Neth J Med 56: 25-31.

13. Paes ML, Powell H (1994) Chylothorax: an update. Br J Hosp Med 51: 482-490.

14. Blalock A, Cunningham RS, Robinson CS (1936) Experimental production of chylo-thorax by occlusion of the superior vena cava. Ann Surg 104: 359-364.

15. Sharkey AJ, Rao JN (2012) The Successful use of octreotide in the treatment of traumatic chylothorax. Texas Heart Institute Journal 39: 428-430.

16. McGrath EE, Blades Z, Anderson PB (2010) Chylothorax: Aetiology, diagnosis and therapeutic options. Respir Med 104: 1-8.

17. http://www.uptodate.com/contents/management-of-chylothorax.

18. Mares DC, Mathur PN (1998) Medical thoracoscopic talc pleurodesis for chylothorax due to lymphoma: a case series. Chest 114: 731-735.
19. Cope C, Kaiser LR (2002) Management of unremitting chylothorax by percutaneous embolization and blockage of retroperitoneal lymphatic vessels in 42 patients. J Vasc Interv Radiol 13: 1139-1148

20. Boffa DJ, Sands MJ, Rice TW, Murthy SC, Mason DP, et al. (2008) A critical evaluation of a percutaneous diagnostic and treatment strategy for chylothorax after thoracic surgery. Eur J Cardiothorac Surg 33: 435-439.

21. Litherland B, Given M, Lyon S (2008) Percutaneous radiological management of high-output chylothorax with CT-guided needle disruption. J Med Imaging Radiat Oncol 52: 164-167.

22. Cerfolio RJ, Allen MS, Deschamps C, Trastek VF, Pairolero PC (1996) Postoperative chylothorax. J Thorac Cardiovasc Surg 112: 1361-1365.

23. Diederick WDB, Nigel M, Stuart ML (2015) Interventional Radiologic Management for Chylothorax .Clinical Pulmonary Medicine: 22: 25-30.

24. Stringel G, Teixeira JA (2000) Thoracoscopic Ligation of the Thoracic Duct. JSLS 4: 239-242.

25. Khiatani V, Isaacson A, Yu H, Stavas J (2013) Interventional Radiologic Placement of Denver Pleuroperitoneal Shunt for Refractory Chylothorax. J Vasc Interv Radiol 24: 1073-1074.

26. Sasha D Adams (2014) Chylothorax Treatment and Management. 